\title{
COVID-19 or not COVID-19, that is the question. The role of screening criteria and point-of-care lung ultrasound in the triage decision-making process in an Emergency Department during the phase 2 of the COVID-19 Italian epidemic
}

\author{
Erika Poggiali, Davide Bastoni, Mariachiara Ferrari, Dario Moretto, Federico Buttafava, Pau \\ Mateo Ramos, Valentina Burzio, Jolanda Petri, Andrea Magnacavallo, Andrea Vercelli
}

Emergency Department, “Guglielmo da Saliceto” Hospital, Piacenza, Italy

\begin{abstract}
In the COVID-19 era the real challenge for the Emergency Departments (ED) is to avoid the spread of the viral infection within the so called "clean area" of the emergency room and the hospital. Different protocols have been proposed and adopted in the EDs to quickly identify suspected COVID-19 patients and to correctly
\end{abstract}

Correspondence:Erika Poggiali, Emergency Department, "Guglielmo da Saliceto" Hospital, Via Giuseppe Taverna 49, Piacenza, Italy. Tel.: +390523 303044

E-mail: erikapoggiali2@gmail.com

Key words: COVID-19, lung ultrasound, Italian epidemic, triage, Emergency Department.

Acknowledgements: The authors are grateful to all the medical staff of their hospital, especially the three bed managers, Belinda Riboni, Damiana Muroni and Gianluca Lisè, for their precious help to find the best possible solution every day in such a difficult emergency.

Contributions: All authors contributed equally to the design of the study, the draft and critical review of the paper. All authors moreover approved the final version and stated the integrity of the whole work.

Conflicts of interest: None. This work was not supported by any grant. EP is a member of the editorial board of Emergency Care Journal.

Availability of data and materials: All data generated or analyzed during this study are included in this published article.

Ethics approval and consent to participate: The study was approved by the local Ethics Committee, "Comitato Etico AVEN dell'Area Vasta Emilia Nord", Protocol Number 2020/60883, Registry Number $601 / 2020 /$ OSS. No animal was used for research proposal. Data was collected without patient identifiers, patients' consent to participate was not required under our hospital's Institutional Review Board guidelines.

Consent for publication: Not applicable.

Received for publication: 26 February 2021.

Revision received: 3 April 2021.

Accepted for publication: 6 April 2021.

This work is licensed under a Creative Commons Attribution 4.0 License (by-nc 4.0).

COpyright: the Author(s), 2021

Licensee PAGEPress, Italy

Emergency Care Journal 2021; 17:9708

doi:10.4081/ecj.2021.9708 manage these patients, all based on clinical and epidemiological criteria. To the best of our knowledge, our pre-triage decisional making-process first integrates the pre-triage interview with pointof-care Lung Ultrasound (LUS) performed in the triage area. The aim of our study is to assess the sensitivity and specificity of our screening clinical and/or epidemiological criteria, and to investigate the role of LUS in the triage decision-making process during the "phase 2" of the COVID-19 Italian epidemic. Our study confirms the pivotal role of the triage in the decision-making process and the management of the entire ED, and it demonstrates that further studies are necessary to validate the role of LUS as tool to promptly identify COVID-19 patients, if combined with a correct pre-triage interview.

\section{Introduction}

The spread of coronavirus disease 2019 (COVID-19) has had a deep impact on the Italian healthcare system, forcing a dramatic and rapid change in the working daily routine of the Emergency Departments (ED). New roles and skills have been proposed to manage both the great influx of patients and to avoid the spread of the viral infection within the so called "clean area".,

Piacenza (Emilia-Romagna, Northern Italy) has reached the peak of COVID-19 epidemic into two weeks, between $9^{\text {th }}$ to $23^{\text {rd }}$ March 2020. In this period, the so called "phase 1", quite all the accesses to the ED of the "Guglielmo da Saliceto" hospital were due to COVID-19. To avoid the complete collapse of our ED because of the high influx of critically ill COVID-19 patients, we developed a long-term plan against catastrophic consequences and to rearrange the patients' pathway throughout our ED. We planned a deep structural and environmental re-organization of the ED, ${ }^{3,4}$ and designed a survival strategy based on a clear triage process starting from the strictly collaboration between the triage nurse, who interviewed the patient, and the emergency clinician, who performed the point-of-care Lung Ultrasound (LUS) in a "key area" of the triage room, in order to quickly identify ultrasound signs of pulmonary interstitial syndrome. In our experience, we confirmed the role of LUS as a safe, easy and sensitive imaging technique to assess the lung and pleural involvement in COVID-19 pneumonia, ${ }^{5,6}$ as reported in literature. ${ }^{7-11}$ In our experience, during the "phase 1" of the Italian COVID-19 epidemic, LUS has been a powerful method to early detect lung damage even in patients who did not complain of respiratory symptoms and/or fever. ${ }^{12}$ Our results highlighted and confirmed the role of LUS in the triage making-decision process, and its pivotal role in the "phase 1" to avoid the viral spread within the "clean area" of the ED and the "clean wards" of the hospital. ${ }^{2}$ After the epidemic phase, in the so 
called "phase 2", since April 1 $1^{\text {st }}, 2020$ we observed an increasing number of patients referred to our ED for no-COVID-19 related complaint. This evidence has changed again our perspectives, causing a new challenge for the organization of our ED: to discriminate a "COVID-19 pathway" from a "COVID-19 free pathway" from the door of the ED to avoid the spread of the virus in the ED, and therefore, in all the hospital. To the best our knowledge, the protocols adopted in the EDs to quickly identify suspected COVID-19 patients and to correctly manage these patients, were all based on clinical and/or epidemiological criteria. ${ }^{13-16}$ None of these studies included LUS as a part of the triage decision-making process.

The aim of our study is to assess the sensitivity and specificity of our screening clinical and epidemiological criteria in detecting COVID-19 patients in the pre-triage area, and to investigate the role of LUS in the triage making-process during the "phase 2" of the COVID-19 Italian epidemic.

\section{Materials and Methods}

This is a retrospective observational study including 222 patients (115 males; 107 females) consecutively admitted to the ED of "Guglielmo da Saliceto" Hospital in Piacenza, EmiliaRomagna, Italy, from April $1^{\text {st }}$ to April $7^{\text {th }}, 2020$. The study was approved by the local ethics committee.

\section{Organization of the ED and pre-triage interview}

During the phase 2 of the COVID-19 Italian epidemic, we decided to split the ED into two areas to avoid the spread of the coronavirus in our ED: a "COVID-19 area" and a "COVID-19 free area". Each area had a dedicated and independent medical and nursing team, supplied with a LUS station. In both areas, medical and nursing staff wore proper Personal Protective Equipment (PPE) as follows: FFP2 or FFP3 mask, protective goggles or face shields, long-sleeved disposable gown (COVID-19 area) or plastic apron (COVID-19 free area), and double gloves.

Before being admitted to the ED, all the patients were interviewed by an expert nurse in the pre-triage area, located outside the hospital, close to the entrance of the ED, using a standard schedule to investigate clinical and epidemiological criteria for COVID-19.

When evaluated by the local pre-hospital Emergency Medicine System (EMS), the medical or nursing team had to fill in the same schedule adopted in the pre-triage area for each patient before their arrival to the emergency room.

Clinical criteria were considered positive in presence of one of the following conditions: i) at least one of the following symptoms in the previous 14 days: fever, cough, nausea or vomiting, diarrhea, abdominal pain; ii) respiratory failure at admission, defined as $\mathrm{SpO} 2<95 \%$ at room ambient or respiratory rate $>25$ acts per minute; iii) a known positive real-time polymerase chain reaction (RT-PCR) nasopharyngeal swab for SARS-CoV-2.

Epidemiological criterion resulted positive if the patient has had a close contact in the last 14 days with a subject with a positive nasopharyngeal swab for SARS-CoV-2.

In the pre-triage area, all the patients had to wear a surgical mask and disinfect their hands with an alcoholic solution. Body temperature and respiratory parameters (peripheral blood saturation, $\mathrm{SpO} 2$ - and respiratory rate) were always checked by the nurse and reported in the pre-triage schedule.

\section{Lung Ultrasound (LUS)}

We investigated all the patients using a rapid and simple lung ultrasound (LUS) protocol to explore all the pulmonary fields from the apex to the bases, both anterior and posterior, as follows: 2 anterior, 2 posterior and 2 lateral (total 12 areas). LUS was performed in the triage area using a portable ultrasound machine ESAOTE Mylab XPro30 equipped with a convex probe CA631 (frequency range 1-8 MHz) (ESAOTE Medical Systems, Florence, Italy). All the LUS were performed by a member of the medical team of our ED, including who were considered inexperienced in LUS, i.e. with less than 6 months of experience. Based on the observation that each pulmonary area should be scanned for 10 seconds, the mean time to perform a complete and correct LUS was 2 minutes. LUS was considered positive in presence of B-pattern (interstitial syndrome with B-lines in clusters, both with three or more separate or coalescent B-lines, and white lung) with spared areas in at least two fields for each hemithorax, as reported in the literature. ${ }^{17}$

To direct the patient to the correct area, we integrated the results of the pre-triage interview and LUS, as follows: i) if the epidemiological criterion or LUS were positive, the patient was admitted to the COVID-19 area; ii) if the clinical criteria were positive for fever and/or cough, the patient was admitted to the COVID-19 area; iii) if the clinical criteria were positive for nausea or vomiting, diarrhea, abdominal pain or acute respiratory failure $(\mathrm{SpO} 2<95 \%)$ with negative LUS, the patient was admitted to the COVID-19 free area; iv) if all the criteria (clinical, epidemiological and LUS) were negative, the patient was admitted to the COVID-19 free area.

\section{Study participants}

We enrolled all the adult patients ( $>13$ years old) admitted to the ED of Guglielmo da Saliceto Hospital, Piacenza, Italy, from April $1^{\text {st }}$ to April $7^{\text {th }}, 2020$, during the so called "phase 2" of the Italian COVID-19 epidemic. The patients' enrollment has been limited only to daytime ( 8 a.m. -8 p.m.) due to the need to perform LUS in the triage area. For the limited medical resources during COVID-19 epidemic, the presence of an "extra" physician in the triage area could be guaranteed only during daytime.

\section{Classification of the patients and data collection}

All the data (clinical and epidemiological criteria, LUS, radiological and laboratory findings including RT-PCR nasopharyngeal swab) were collected anonymously in a database.

RT-PCR nasopharyngeal swab was performed in all the patients to be admitted, or in those with a strong clinical suspicious of SARS-CoV-2 infection. In our experience, a positive nasopharyngeal swab is sufficient to define the patient as "positive", but a negative nasopharyngeal swab in presence of highly suspected clinical and radiological criteria cannot exclude COVID-19. ${ }^{18}$ To clearly define a patient as "negative", a negative RT-PCR nasopharyngeal swab is mandatory but not sufficient, due to the high frequency of false negative and asymptomatic carriers.

As consequence, we defined as "positive" for COVID-19, a patient with: i) a RT-PCR nasopharyngeal swab positive for SARSCoV-2 at admission in the ED or within 72 hours, or ii) a clinical criterion and signs of interstitial pneumonia at chest X-Ray or high-resolution CT-scan even with negative RT-PCR nasopharyngeal swab.

We defined as "negative", a patient with a negative RT-PCR nasopharyngeal swab at admission in the ED or within 72 hours, and: i) neither clinical nor epidemiological criteria nor radiological signs of COVID-19 pneumonia, or ii) the absence of antibodies 
(IgG) against SARS-CoV-2 in a period between 1 and 3 months after discharge.

We defined "indeterminate" a patient who did not meet neither the positive nor the negative criteria.

\section{Outcome}

The primary outcome of the study is to determine the different sensitivity and specificity for COVID-19 infection of the pre-triage interview alone or associated with LUS.

\section{Statistical analysis}

We analysed the patients classified as positive or negative according to the adopted criteria. We calculated sensitivity, specificity, positive and negative predictive values of the triage interview with or without LUS in detecting COVID-19 patients. We used $95 \%$ confidence intervals (CI) to compare the two groups.

\section{Results}

Two hundred twenty-two consecutive patients were enrolled in the study: 115 (52\%) males, 107 (48\%) females. The mean age was 59.7 years (ranging from 15 to 99 ).
Based on the criteria used to define a patient as positive or negative, 148/222 (67\%) patients have been classified correctly. Seventy-four (34\%) patients were considered as indeterminate and excluded from statistical analysis (Figure 1). LUS was not performed in 39/148 patients.

LUS was performed in 109/148 (73.6\%) patients with a defined diagnosis. These patients have been considered for the statistical analysis: 37/109 (34\%) were "positive" (infected) cases and $72 / 109(66 \%)$ were "negative" (not infected) cases. Among the positive cases, 27/37 had a positive nasopharyngeal swab. Ten COVID-19 patients had a negative nasopharyngeal swab: 4/10 patients have been investigated with LUS, that resulted as positive. Chest HRTC has been performed in the emergency room in all the patients and confirmed the diagnosis of COVID-19 pneumonia.

In the Table 1 we reported the clinical characteristics of the 109 investigated patients at presentation in the triage area. The degree of acute respiratory failure has been based on the patient's oxygen need at admission. The expert triage nurse treated immediately the patient's respiratory failure with oxygen therapy in the triage area, based on peripheral blood oxygen saturation using different oxygen flows, as reported in Table 1.

The following $2 \times 2$ contingency tables (Table 2, 3 and 4 ) sum-

222 enrolled patients

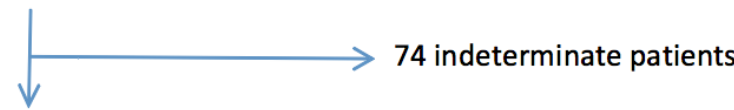

148 patients with a defined diagnosis (positive/negative)

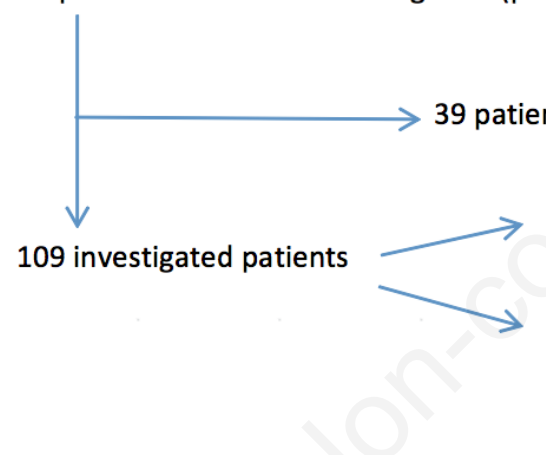

25 patients in the COVID-19 area

49 patients in the free COVID-19 area

Figure 1. Flow-chart of the enrolled (222) and investigated (109) patients of the study. NP, nasopharyngeal. LUS, lung ultrasound.

Table 1. Patients' demographic and clinical characteristics at presentation in the ED. M, male; F, female. Respiratory failure has been classified according to the patient's oxygen need at admission in the triage as follows: mild till $6 \mathrm{LT} / \mathrm{min}$ in nasal cannulae; moderate for flow rate of $10 \mathrm{LT} / \mathrm{min}$ with non-rebreather mask; severe for flow of at least $15 \mathrm{LT} / \mathrm{min}$ with non-rebreather mask.

\begin{tabular}{lcc} 
& COVID19 patients (n 37) & Not COVID19 patients (n 72) \\
Sex (M/F) & $21 / 16$ & $41 / 31$ \\
Mean age (years) (min-max) & $69(19-96)$ & $61(16-95)$ \\
\hline fever & 20 & 12 \\
Respiratory symptoms & 15 & 9 \\
Gastrointestinal symptoms & 9 & 8 \\
\hline Acute respiratory failure & 17 & 6 \\
Mild & 8 & 4 \\
Moderate & 3 & 1 \\
Severe & 6 & 1 \\
\hline
\end{tabular}


marize the results of the pre-triage interview, LUS and the two integrated tests. A positive test corresponds to the assignment to the COVID-19 area, while a negative test to the COVID-19 free area.

The performance of the pre-triage interview and LUS were evaluated for sensitivity, specificity, and Positive (PPV) and Negative Predictive Value (NPV), as reported in Table 5.

The pre-triage interview alone has a better sensitivity than combined with LUS: respectively, 97.3\% (95\% CI, 94-100) versus $94.6 \%$ (95\% IC, 88-100), but the difference is not statistically significant. Even the specificity of the pre-triage interview alone and combined with LUS are not statistically significant: they are respectively $66.7 \%$ (95\% IC, 56-78) and 69.4\% (95\% IC, 59-79). Interestingly the specificity of LUS is $77.8 \%$ (95\% IC, 70-86), but this result is not statistically significant.

LUS resulted as negative in 9/37 (24\%) COVID-19 patients: they complained upper respiratory symptoms (2/9), severe bilateral pleural effusion (2/9), nausea (1/9), anemia (1/9), diarrhea (1/9), palpitation (1/9) and acute chest pain (1/9). Only 2 out of these patients were addressed to the free COVID-19 area (false negative): one patient with a final diagnosis of anemia had a negative pre-triage interview and a negative LUS with no signs of pneumonia at chest X-ray; the other had a severe heart failure with massive bilateral pleural effusion. Based on LUS, the patient was addressed to the COVID-19 free area. Chest X-ray confirmed the diagnosis of congestive heart failure. Both the patients can be considered "asymptomatic carriers".

Twelve out 14 COVID-19 negative patients referred to the ED for nausea, vomiting, diarrhea, abdominal pain or respiratory failure (see Table 1) were correctly addressed to the COVID-19 free area based on negative LUS. In our experience, 9/72 (12\%) no COVID-19 patients were addressed to COVID-19 area based only on LUS.

Considering the patients with respiratory failure (23/109, $21 \%$ ), both infected and not infected for COVID-19 (see Table 1), LUS has a sensitivity and specificity of $100 \%$ (95\% IC, 98-100) in patients with severe respiratory failure. In patients with mild and moderate respiratory failure, the specificity of LUS is of $0 \%$, while the sensitivity is respectively of $87.5 \%$ (95\% IC, 85-89) and $66.7 \%$ (95\% IC, 62-71). Since all the patients were investigated with the pre-triage interview, we calculated sensitivity and specificity of the test also for all the patients with a defined diagnosis (148/222), resulting respectively $96.8 \%$ and $65.1 \%$. Among them, 60 patients resulted true positive, 2 false negative, 56 true negative and 30 false positive. Among the patients who were not investigated with LUS (39/148, 26.4\%), 25/148 (13\%) have been resulted positive. Among them, 17 patients were addressed to COVID-19 area for both clinical and epidemiological criteria at the pre-triage interview: LUS would not change the final destination of the patients, i.e. COVID-19 area. If we consider the inderminate group, 25/74

Table $2.2 \times 2$ contingency table for the pre-triage interview.

\begin{tabular}{lcccc} 
& $\mathbf{N}^{\circ}$ of infected patients & $\mathbf{N}^{\circ}$ of non-infected patients & Total & 60 \\
Positive pre-triage interview & 36 & 24 & 48 \\
Negative pre-triage interview & 1 & 72 & 49 \\
\hline Total & 37 & 72 & \\
\hline
\end{tabular}

Table 3. $2 \times 2$ contingency table for LUS.

\begin{tabular}{lcccc} 
& $N^{\circ}$ of infected patients & $\mathbb{N}^{\circ}$ of non-infected patients & Total & 44 \\
Positive LUS & 28 & 16 & 56 & 65 \\
Negative LUS & 9 & 72 & 56 \\
\hline Total & 37 & 72 & \\
\hline
\end{tabular}

Table 4. $2 \times 2$ contingency table for the integrated approach: the pre-triage interview and LUS.

\begin{tabular}{lcccc} 
& $\mathbb{N}^{\circ}$ of infected patients & $\mathbb{N}^{\circ}$ of non-infected patients & Total & 57 \\
Positive interview + LUS & 35 & 22 & 52 \\
Negative interview + LUS & 2 & 50 & 72 \\
\hline Total & 37 & 72 & 5 \\
\hline
\end{tabular}

Table 5. Sensitivity, specificity, Positive and Negative Predictive Value (PPV, NPV) of the pre-triage interview, LUS and the combination of both the strategies.

\begin{tabular}{lcccc} 
& Sensitivity (\%) & Specificity (\%) & PPV (\%) NPV (\%) \\
Pre-triage interview & 97.3 & 66.7 & 60.0 & 97.9 \\
LUS & 75.7 & 77.8 & 63.6 & 86.1 \\
\hline Pre-triage interview + LUS & 94.6 & 69.4 & 61.4 & 96.1 \\
\hline
\end{tabular}


(34\%) patients have been addressed to the COVID-19 area: in 8 patients we did not perform a nasopharyngeal swab because they had a positive epidemiological criterion in absence of symptoms suspected for COVID-19, and none of them were hospitalized. Forty-nine out of seventy-four (66\%) patients have been assigned to the COVID-19 free area: none of them had a nasopharyngeal swab in absence of clinical and epidemiological criteria and they have been all discharged home from the ED.

\section{Discussion}

The real challenge for the ED is to identify COVID-19 patients from their arrival at the ED. Unfortunately, SARS-CoV-2 is the cause of an infection, which can run its course without symptoms (asymptomatic carrier) or with a very broad spectrum of symptoms, ranging from fatigue, dry cough, fever, gastrointestinal symptoms, anosmia or dysgeusia to acute respiratory distress syndrome with a high risk of death for multiorgan failure. ${ }^{12,19-21}$ In the pandemic phase of COVID-19, the most common cause of access to our ED was dyspnea of different severity, but we also observed a significant number of patients with gastrointestinal symptoms even in absence of fever and/or dyspnea. ${ }^{12}$

The main purpose of our study was to design a triage makingprocess to avoid the spread of the infection in the COVID-19 free area due to misdiagnoses. Our study demonstrated a prevalence of the disease of $42 \%$ in the sample of patients with a defined diagnosis $(62 / 148)$. Unfortunately, a rapid and reliable test to identify COVID-19 patients in the pre-triage process is still unavailable. For this reason, we decided to give preference to sensitivity of our protocol strategy with the aim of losing as few positive cases as possible, reducing the risk of transmitting infection to non-infected patients.

Based on our previous experience, we decided to investigate in the pre-triage interview not only fever and respiratory symptoms, but also gastrointestinal symptoms to avoid and limit misdiagnoses. This led us to reach a sensitivity of $97.3 \%$ and a negative predictive value of $97.9 \%$ for the pre-triage interview. If we consider the sample of all the patients with a defined diagnosis $(148 / 222)$, the sensitivity is of $96.8 \%$ due to two false negative patients, and the predictive negative value of $96.5 \%$. The main limit of the pre-triage interview is the low specificity: of $66.7 \%$ in the analyzed sample (109/222), and of $65 \%$ in the overall sample with a defined diagnosis (148/222), and as consequence, the high risk of targeting incorrectly uninfected patients in the COVID-19 area: 24/60 (40\%) patients addressed to COVID-19 area have been resulted negative. To try to solve this important limitation, we evaluated all the patients with LUS in the triage area. LUS was used with two main aims: to increase the sensitivity and specificity of the pre-triage interview, respectively through the identification of COVID-19 patients who did not complain of fever and respiratory symptoms, and the diagnosis of alternative diseases in case of respiratory failure, such as congestive heart failure, pneumothorax, or reactivation of chronic obstructive pulmonary disease. Our results demonstrated that LUS has a lower sensitivity compared to the pre-triage interview (respectively, 75.7\% versus 97.3\%), but a higher specificity (respectively, $77.8 \%$ versus $66.7 \%$ ), even if not statistically significant. We are aware that the low sensitivity of LUS is related to the identification of radiological findings of a complication, i.e. COVID-19 related pneumonia, that can be absent at diagnosis and usually develops after 9 days from the infection, approximately 3-7 days after the emergence of clinical signs and symptoms, as reported for chest CT-scan. ${ }^{22}$

Considering only the patients with respiratory failure, we reported surprisingly different results. In the clinical scenario of COVID-19 pandemic, a patient with severe acute respiratory failure related to SARS CoV2 infection has surely LUS signs of pneumonia. As consequence, in this subgroup of patients, LUS has a sensitivity significantly higher compared to the population of the study. On the contrary, the significant difference of the specificity of LUS in patients with severe vs mild-moderate respiratory failure can be related to the expertise of the emergency clinician. In this context with a high prevalence of COVID-19 pneumonia, emergency clinicians can be led to overestimate pulmonary involvement.

Based on all these observations, we promptly interrupted the study after only one week, and we created a new pre-triage protocol with a third pathway, so called "intermediate", and we also added three new criteria for suspected patients, including: anosmia or dysgeusia in the last 20 days; or at least two of these symptoms: headache, myalgia, joint pain, conjunctivitis; or $\mathrm{SpO} 2<92 \%$ at room ambient for patients with COPD. All the patients classified as "intermediate" by the pre-triage nurse, were addressed and evaluated in a new dedicated area of the ED called "intermediate area".

We are aware that the main limits of our study are the small size of the patients' sample and the difficulty to integrate LUS and the pre-triage interview. In addition, we did not routinely perform a nasopharyngeal swab in patients sent to the COVID-19 free area.

Anyway, starting from the strong conviction that further evidence is needed to confirm or disprove the role of LUS in the triage decision-making process in the phase 2 of the pandemic, we continued to evaluate all the patients with LUS, and we introduced in the working algorithm the so called "LUS score" based on the lung involvement for each of the 12 fields ( 2 anterior, 2 posterior and 2 lateral), as follows: 0 - normal; 1 - B lines $\geq 3$ in a field; 2 - "white lung"; 3 - consolidations. ${ }^{8}$ We created a score based on the integration of LUS and the pre-triage interview to correctly address the patients to the three different area of the ED: a clean area, an infected area and an indeterminate area. Preliminary results confirm our hypothesis (data not shown).

\section{Conclusions}

Our study confirmed the pivotal role of the triage in the management of a medicine disaster scenario. ${ }^{23}$ The study failed to demonstrate the utility of LUS in the triage setting, but we think that the small size of the patients' sample is the main limit of our work and it surely affects the results. For this reason, we believe that further studies are necessary to validate the role of LUS as tool to promptly identify COVID-19 patients if combined with the pretriage interview. Our data and experience suggest that is not possible to surely identify COVID-19 patients in the triage area in all the cases. As consequence, unrecognized positive patients can be addressed to the "COVID-19 free area" and put at risk of infection negative patients. Based on our results, we believe that an "intermediate area" is necessary to limit the contamination of the COVID-19 free area. We are aware that every change in the organization of the ED is a great challenge considering the limited resources, and it involves a deep effort of all the medical and nursing staff, but we strongly believe that the pre-triage protocol remains the most powerful tool to avoid the spread of the virus in the ED and in the hospital, particularly in the phase 2 of the pandemic. 


\section{References}

1. Comelli I, Scioscioli F, Cervellin G. Impact of the covid-19 epidemic on census, organization and activity of a large urban emergency department. Acta Biomed 2020;91:45-9.

2. Erika P, Andrea V, Cillis MG, et al. Triage decision-making at the time of COVID-19 infection: the Piacenza strategy. Intern Emerg Med 2020;15:879-82.

3. Poggiali E, Vercelli A, Mazzoni S, et al. COVID-19 pandemic, piacenza calling. The survival strategy of an Italian emergency department. Acta Biomed 2020;91:1-3.

4. Maniscalco P, Poggiali E, Quattrini F, et al. The deep impact of novel covid-19 infection in an Orthopedics and traumatology department: The experience of the Piacenza hospital. Acta Biomed 2020;91:97-105.

5. Dacrema A, Silva M, Rovero L, et al. A simple lung ultrasound protocol for the screening of COVID-19 pneumonia in the emergency department. Intern Emerg Med 2021. doi:10.1007/s11739-020-02596-6. Online ahead of print.

6. Poggiali E, Dacrema A, Bastoni D, et al. Can Lung US Help Critical Care Clinicians in the Early Diagnosis of Novel Coronavirus (COVID-19) Pneumonia? Radiology 2020:200847.

7. Fiala MJ. A Brief Review of Lung Ultrasonography in COVID-19: Is It Useful? Ann Emerg Med 2020;75:784-5.

8. Soldati G, Smargiassi A, Inchingolo R, et al. Proposal for International Standardization of the Use of Lung Ultrasound for Patients With COVID-19. J Ultrasound Med 2020;39:1413-9.

9. Vetrugno L, Bove T, Orso D, et al. Our Italian experience using lung ultrasound for identification, grading and serial follow-up of severity of lung involvement for management of patients with COVID-19. Echocardiography 2020;37:625-7.

10. Sorlini C, Femia M, Nattino G, et al. The role of lung ultrasound as a frontline diagnostic tool in the era of COVID-19 outbreak. Intern Emerg Med 2020. doi:10.1007/s11739-02002524-8

11. Buonsenso D, Piano A, Raffaelli F, Bonadia N, de Gaetano Donati K, Franceschi F. Point-of-care lung ultrasound findings in novel coronavirus disease-19 pnemoniae: A case report and potential applications during COVID-19 outbreak. Eur Rev
Med Pharmacol Sci 2020;24:2776-2780.

12. Poggiali E, Mateo Ramos P, Bastoni D, et al. Abdominal pain: a real challenge in novel COVID-19 infection. Eur J Case Rep Intern Med 2020;7:001632.

13. Carenzo L, Costantini E, Greco M, et al. Hospital surge capacity in a tertiary emergency referral centre during the COVID19 outbreak in Italy. Anaesthesia 2020;75:928-34.

14. Levy Y, Frenkel Nir Y, Ironi A, et al. Emergency Department Triage in the Era of COVID-19: The Sheba Medical Center Experience. Isr Med Assoc J 2020;22:470-5.

15. Turcato G, Zaboli A, Pfeifer N. The COVID-19 epidemic and reorganisation of triage, an observational study. Intern Emerg Med 2020;15:1517-1524.

16. Wee LE, Fua TP, Chua YY, et al. Containing COVID-19 in the emergency department: the role of improved case detection and segregation of suspect cases. Acad Emerg Med 2020;27:379-87.

17. Volpicelli G, Gargani L. Sonographic signs and patterns of COVID-19 pneumonia. Ultrasound J 2020;12.

18. Poggiali E, Vercelli A, Vadacca GB, et al. Negative nasopharyngeal swabs in COVID-19 pneumonia: The experience of an italian emergency department (Piacenza) during the first month of the italian epidemic. Acta Biomed 2020;91:1-4.

19. Chen N, Zhou M, Dong X, et al. Epidemiological and clinical characteristics of 99 cases of 2019 novel coronavirus pneumonia in Wuhan, China: a descriptive study. Lancet 2020;395:507-13.

20. Huang C, Wang Y, Li X, et al. Clinical features of patients infected with 2019 novel coronavirus in Wuhan, China. Lancet 2020;395:497-506.

21. Pan F, Ye T, Sun P, et al. Time course of lung changes at chest CT during recovery from Coronavirus disease 2019 (COVID19). Radiology 2020;295:715-21.

22. Chakraborty C, Sharma AR, Sharma G, Bhattacharya M, Lee SS. SARS-CoV-2 causing pneumonia-associated respiratory disorder (COVID-19): Diagnostic and proposed therapeutic options. Eur Rev Med Pharmacol Sci 2020;24:4016-26.

23. Nakao H, Ukai I, Kotani J. A review of the history of the origin of triage from a disaster medicine perspective. Acute Med Surg 2017;4:379-84. 